\title{
HEAT TRANSFER ANALYSIS FOR LI-ION BATTERY PACKAGE WITH A HYBRID THERMAL MANAGEMENT SYSTEM USING PHASE CHANGE MATERIAL AND FORCED CONVECTION
}

\author{
JOON AHN ${ }^{1}$, JEONG CHUL SONG ${ }^{2} \&$ JOON SIK LEE ${ }^{3}$ \\ ${ }^{1}$ School of Mechanical Engineering, Kookmin University, South Korea \\ ${ }^{2}$ Samsung Electronics, South Korea \\ ${ }^{3}$ School of Mechanical and Aerospace Engineering, Seoul National University, South Korea
}

\begin{abstract}
Battery charging and performance maintenance is one of the core technologies of electric vehicles. The performance of the battery is temperature-sensitive and the temperature should be kept within a certain range. Due to the nature of the car being driven and parked in the outdoors with extreme temperature changes, an efficient thermal management system is required. Cooling fans are mainly used for thermal management, but the power to drive the fans is used and the cooling capacity is limited. A method of using phase change materials has been proposed. The phase change material has a disadvantage in that the operating point is limited and heat transfer control is not easy. In this study, we propose a hybrid thermal management system with a cooling fan and a phase change material filled inside the battery pack. In order to investigate the cooling performance of the system, a series of CFD has been conducted for the hybrid cooling unit of the Li-ion battery pack in which the phase change and forced convection by implementing an immersed boundary method handling the conjugate heat transfer with a phase change. The simulation results show that the uniformity of the inter-cell temperature can be greatly improved when the phase-change material is filled.
\end{abstract}

Keywords: Li-ion battery, battery thermal management, phase change material, immersed boundary method.

\section{INTRODUCTION}

Tesla, a recent electric car, is gaining popularity in the United States and major automakers are spurring the development of electric vehicles. In the case of electric vehicles, battery charging and performance maintenance is one of the key technologies. The performance of the battery should be maintained within a certain range because it is sensitive to temperature. It is used in an outdoor environment with a severe temperature change, and an efficient thermal management system is required due to the nature of the automobile normally parked outside [1].

Although the cooling fan is massively used for the thermal management, the power for driving the fan is used and the cooling ability is limited, so that heat transfer enhancement techniques have been studied [2]. The use of phase change materials has also been proposed as an alternative [3], [4]. However, in the case of phase change materials, it is pointed out that the operating point is limited depending on the material and heat transfer control is not easy. In this study, as illustrated in Fig. 1, we propose a hybrid thermal management system that is filled with phase change material inside a battery pack together with a conventional cooling fan.

In this paper, we have investigated the thermal behavior of a forced convection cooling system without phase change material (PCM) and a hybrid system filled with PCM. This requires simultaneous simulation of the solidification and melting of the PCM, the flow of the convective cooling fluid and the conduction of the package material. In order to analyze 


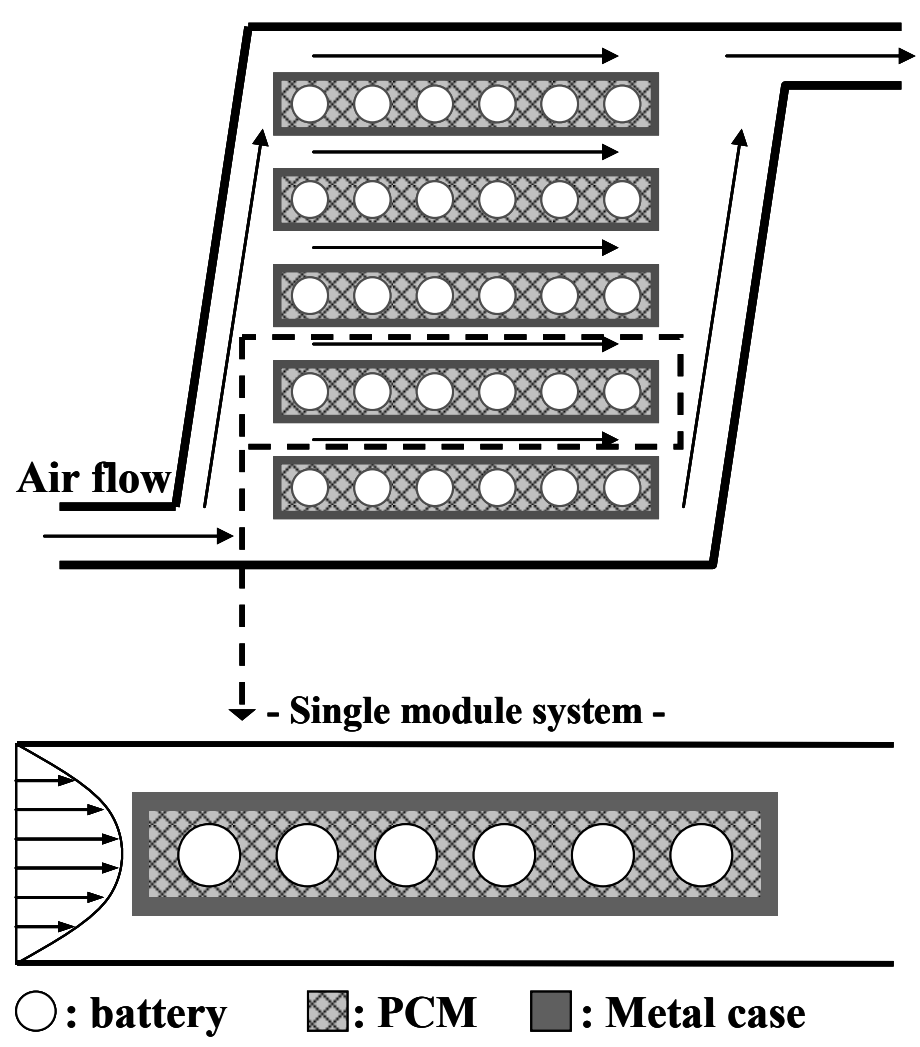

Figure 1: Hybrid battery thermal management system.

the phase change, convection and conduction together, this study extended the immersed boundary method developed for the conjugate heat transfer analysis developed in the previous study [5] to handle the phase change.

In order to verify the newly proposed numerical method, we conducted code validation studies on two cases. As for the conjugate heat transfer in single phase, we have already completed the validation in the previous study, so we conducted numerical simulations for problems including the phase change. First, the one-dimensional two-component phase change problem [6], in which the analytical solution exists, is simulated and compared with the analytical solution. Next, the battery package [3] for the electric vehicle filled with the phase change material was analyzed and compared with the finite element data.

After code validation studies, the hybrid thermal management system of the Li-ion battery package, in which the phase change and the forced convection occur, is simulated. In this study, we designed a package consisting of six Li-ion batteries (Sony type US 18650) as shown in Fig. 1. The temperature of the cooling air was fixed at $30^{\circ} \mathrm{C}$ and the current of the battery was set at $1.8 \mathrm{~A}$. The flow rate of cooling air was determined so that the channel Reynolds number would be 200. Under these conditions, the thermal management system is analyzed when charging and discharging are repeated in 1 hour cycle with the state of charge (SOC) ranging from 0.25 to 0.75 . The performance of the hybrid thermal management system was evaluated by comparing the time to reach the critical temperature and the temperature uniformity among batteries. 


\section{NUMERICAL METHOD}

The non-dimensionalized Navier-Stokes equations and energy equation presented in eqns (1)-(3) are the governing equations in this study

$$
\begin{gathered}
\frac{\partial u_{i}}{\partial x_{i}}-M s=0 \\
\frac{\partial u_{i}}{\partial t}+\frac{\partial u_{i} u_{j}}{\partial x_{j}}=-\frac{\partial p}{\partial x_{i}}+\frac{1}{\operatorname{Re}} \frac{\partial^{2} u_{i}}{\partial x_{j} \partial x_{j}}+f_{i} \\
\frac{\partial \theta}{\partial t}+\omega \frac{\partial\left(u_{j} \theta\right)}{\partial x_{j}}=\frac{1}{\operatorname{Re} \operatorname{Pr}} \frac{K}{C} \frac{\partial^{2} \theta}{\partial x_{j} \partial x_{j}}+\xi
\end{gathered}
$$

As described in the introduction, the immersed boundary method was adopted in this study. $f_{\mathrm{i}}$ in eqn (2) is momentum forcing to satisfy the non-slip boundary conditions at the interface and $M s$ in eqn (1) is the mass source to satisfy global mass conservation. In eqn (2), momentum forcing $f_{\mathrm{i}}$ is also applied to the inside of the solid, which makes the velocity inside the solid be zero. As a result, the energy equation presented in eqn (3) becomes the conduction equation in the solid interior. $K^{*}$ and $C^{*}$ in eqn (3) are effective thermal conductivity and heat capacity, respectively. They are determined to satisfy the continuity of temperature and heat flux at the interface. $\xi$ is a correction term to maintain a second-order accuracy of the solution and $\omega$ is an indicator function to recognize the solid-fluid interface. Detailed derivation and validation results are presented in [5].

Considering the solidification / melting process of the mixture, there are liquid, solid and mushy zones as shown in Fig. 2. Eqn (3) can be expressed as eqns (4)-(6) for each zone

Solid zone $\quad C_{s} \frac{\partial \theta}{\partial t}=\frac{K_{s}}{\operatorname{Re} \operatorname{Pr}} \frac{\partial^{2} \theta}{\partial x_{j} \partial x_{j}}$,

Mushy zone $C_{s} \frac{\partial \theta}{\partial t}+\frac{\Delta C}{\theta_{l}-\theta_{s}} \frac{\partial \theta^{2}}{\partial t}+\frac{H_{s l}}{\theta_{l}-\theta_{s}} \frac{\partial \theta}{\partial t}=\frac{1}{\operatorname{Re} \operatorname{Pr}} \frac{\partial}{\partial x_{j}}\left(\left(K_{\alpha}+K_{\beta} \theta\right) \frac{\partial \theta}{\partial x_{j}}\right)$,

Liquid zone $\quad C_{l} \frac{\partial \theta}{\partial t}=\frac{K_{l}}{\operatorname{Re} \operatorname{Pr}} \frac{\partial^{2} \theta}{\partial x_{j} \partial x_{j}}$.

Eqn (5) for the mushy zone contains a nonlinear term and is linearized to use the implicit scheme. Through the above process, three zones can be handled in a unitary computational domain.

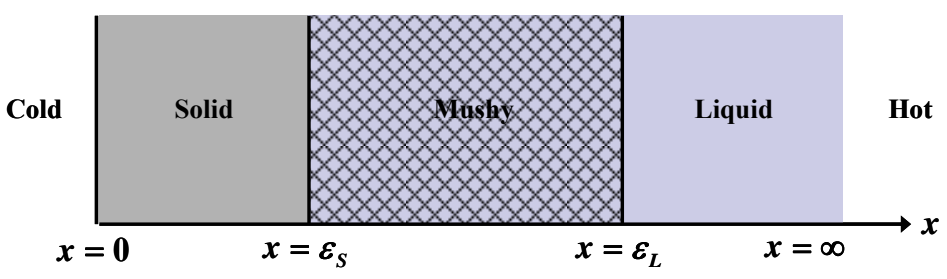

Figure 2: Solidification of a mixture in semi-infinite geometry. 


\section{CODE VALIDATION STUDIES}

The method for the analysis of the heat transfer with phase change is validated against two different problems of conductive heat transfer with phase change, which are the transient conduction with phase change in semi-infinite medium and thermal behavior of battery pack filled with PCM. We first analyzed the solidification problem in the 1-D semi-infinite medium as shown in Fig. 2 with the code developed in this study. This problem has the analytic solution in the form of a similarity solution when defining the similarity variable $\eta=x / \sqrt{t}$ [6]. Fig. 3 compares the simulation results with the analytic solution. Two results are in good agreement.

Next, we analyzed the battery package with PCM shown in Fig. 4(a). The analyzed battery package is a model found in the open literature [3] and contains the same Li-ion battery as adopted in this study. As shown in Fig. 4(a), the convective boundary condition was imposed, where the heat transfer coefficient was set at $1 \mathrm{~W} / \mathrm{m}^{2} \mathrm{~K}$. In [3], the heat generation rate of the battery is modeled by empirical formula as a function of depth of discharge (DoD). The temperature distribution according to DoD is presented in the paper. Hallaj and Selman [3] analyzed the battery the finite element method in a body fitted coordinate system. As shown in Fig. 4(b), the temperature distribution analysed by the immersed boundary method developed in this study agrees well with the finite element data.

\section{HYBRID THERMAL MANAGEMENT SYSTEM}

The hybrid battery thermal management system proposed in this study can be composed of multiple modules as shown in Fig. 1. However, to save computational cost, one module is set as the computational domain as shown in Fig. 5. The Li-ion battery analyzed in this study has a circular cylindrical shape, whose diameter is marked as $D$ in Fig. 5. The module contains six Li-ion batteries inside a stainless steel case with a thickness of $0.1 \mathrm{D}$ and is filled with PCM. Cooling air flows through the outside of the case and the flow rate of the air is set so that the channel Reynolds numbers becomes 200. The inlet temperature of the cooling air was fixed at $30^{\circ} \mathrm{C}$ and a current of $1.8 \mathrm{~A}$ was imposed to flow through the Li-ion battery.

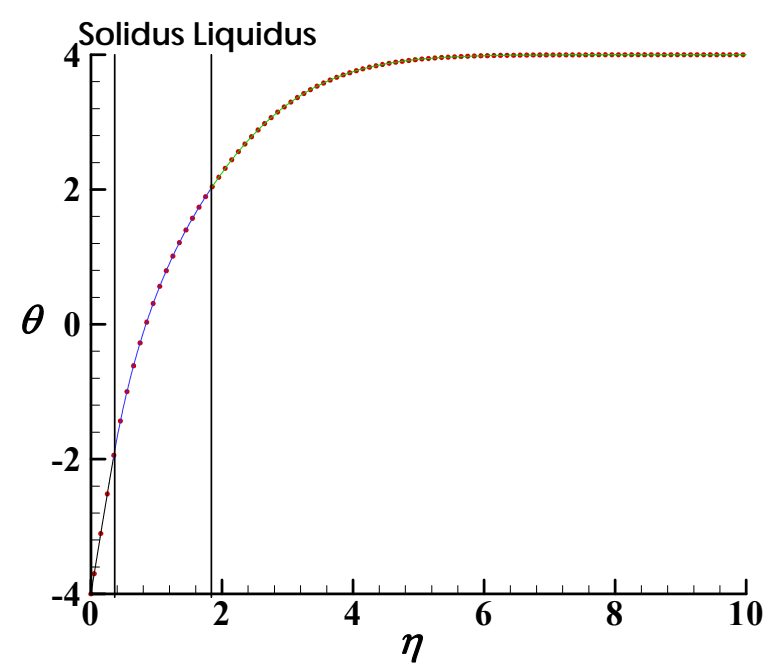

Figure 3: Non-dimensional temperature distribution in a semi-infinite medium with solidification. • Analytic solution; - Numerical data. 
(a) $\quad-k \frac{\partial T}{\partial y}=h\left(T-T_{a t m}\right)$
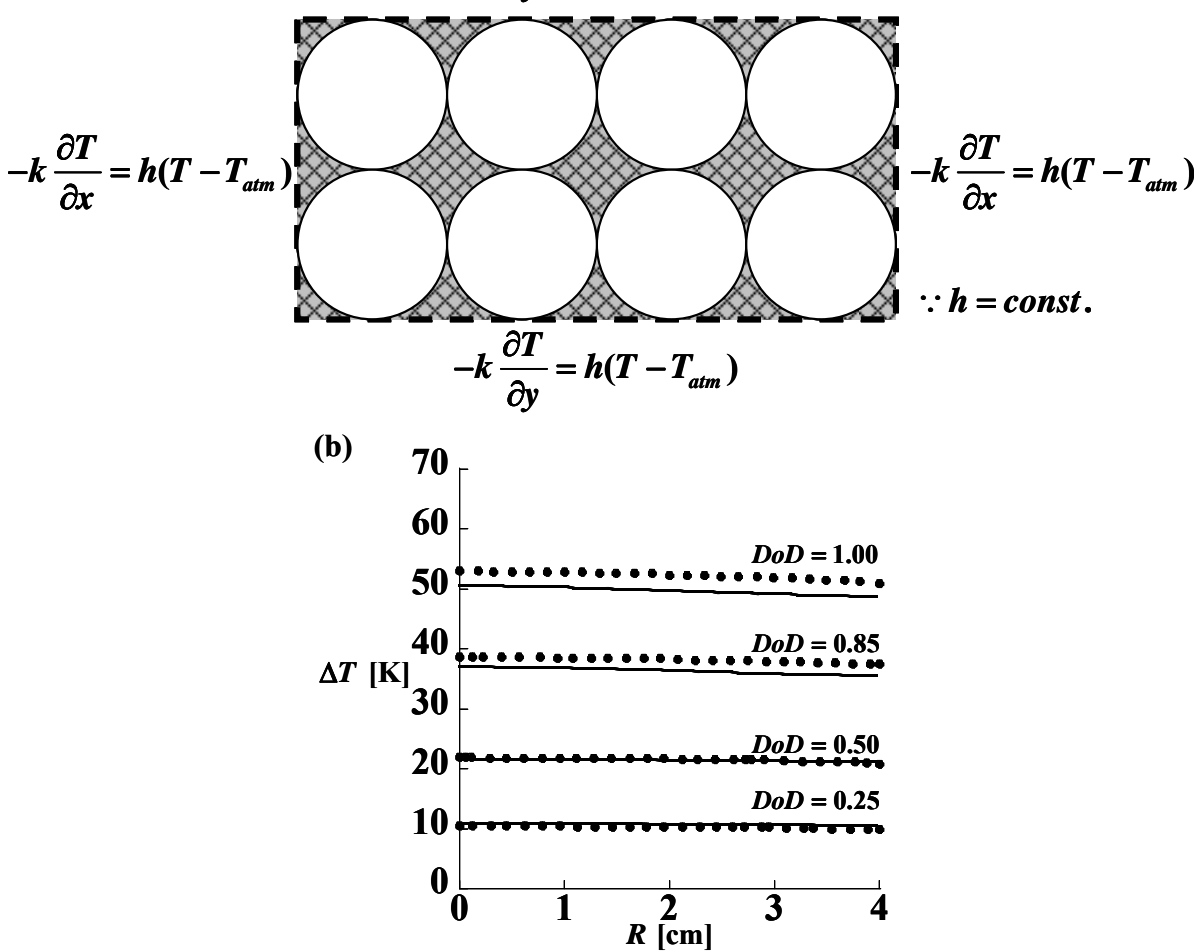

Figure 4: Code validation study on the battery package filled with PCM. (a) Computational domain; (b) Radial temperature distribution in a battery; $\bullet$ Finite element [3]; - Present data.

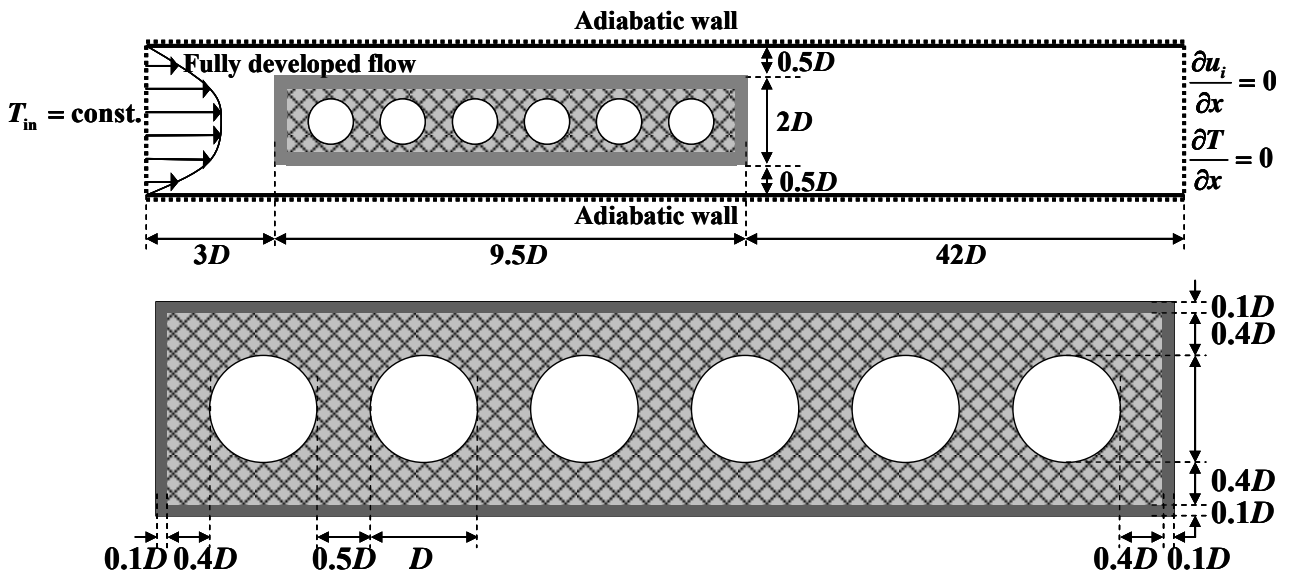

Figure 5: Numerical modelling of the hybrid cooling system ( $D$ : diameter of Li-ion battery). 
The phase change material was assumed to be a commercial product, Rubitherm (RT-24), and the Li-ion battery was assumed to be a Sony type (US 18650). Considering the characteristics of the hybrid vehicle, the SOC is repeated in the range of $0.25-0.75$ in 1 hour cycle. Two - dimensional laminar flow analysis was performed and the exit area of 42D length was set to impose Neumann type boundary conditions.

The initial temperature is $30^{\circ} \mathrm{C}$ and the allowable temperature of the Li-ion battery is $55^{\circ} \mathrm{C}$. Fig. 6 shows the temperature distribution at the point where the critical temperature is reached after starting the simulation. If no PCM is present, the critical temperature is reached in 3.6 hours $\left(t_{\mathrm{cA}, \Delta \mathrm{T}}\right)$. When the PCM is filled, the time to reach the critical temperature is greatly delayed to 12.3 hours $\left(t_{\mathrm{cH}, \Delta \mathrm{T}}\right)$. Since the operating time of a typical automobile does not exceed 12 hours, it is predicted that if the hybrid heat management system is adopted, the temperature of the battery can be managed safely under the conditions of the simulation.

Fig. 6(a) shows the temperature distribution at the point when the critical temperature of the battery package without phase change material reached the critical temperature. At the moment $\left(t_{\mathrm{cA}, \Delta \mathrm{T}}\right)$, the fifth battery located near $x / D=7$ has reached the critical temperature. In the absence of phase change material, the temperature near the stagnation point $(x / D=0)$, which is cooled by external convection and is active in heat transfer, is lowest. As the boundary layer grows on the top and bottom of the case, the heat transfer coefficient decreases and the temperature of the battery gradually rises. The last 6th battery is cooled down also on the back of the case, and its temperature is lower than the 5th battery.

At the same time (3.6 hours after startup), a battery pack filled with phase change material can be observed to be in a safe temperature range (Fig. 6(b)). The overall temperature distribution is similar to that in the absence of phase change materials (Fig. 6(a)) because of the same heat transfer conditions due to external convection, but the overall temperature rise is alleviated and the isothermal spacing is wider. Fig. 6(c) shows the liquid fraction $\left(g_{1}\right)$ of the phase change material at that time. The phase change material is slightly melted downstream but remains in a solid state in most areas and has a significant cooling potential.

When the charge/discharge cycle of the battery is repeated 12.3 times, the battery charged with the phase change material also reaches the critical temperature. If no phase change material is present at this time (Fig. 6(d)), it is predicted that the five batteries, excluding the first battery, will exceed the allowable temperature. It is observed that the battery with PCM is kept at a much lower temperature (Fig. 6(e)) at that time. In the case of a battery packed with PCM, the fifth of the six batteries reached the critical temperature. When we investigate the liquid fraction of the phase change material at this time (Fig. 6(f)), most of the phase change material near the first two batteries remains solid. However, after $x / D=4$ where the third battery is located, it appears to be mushy or liquid.

Fig. 7 shows the temperature changes of the 5th battery with the highest temperature and the first battery with the lowest temperature during the 30 cycles of simulation. The three horizontal lines in Fig. 7 show the allowable temperature of the battery, the melting end temperature of the PCM, and its melting start temperature from the top. The temperatures of the four batteries increase equally at the initial temperature of $30^{\circ} \mathrm{C}$ until they reach the melting start temperature of the PCM. Above the temperature, they tend to be different from each other. The fifth battery of the battery pack without the PCM $\left(T_{\max , A}\right)$ reaches the critical temperature in only 3.6 hours after startup but the fifth battery reaches the critical temperature after 12.3 hours when the PCM is charged $\left(T_{\max H}\right)$. The first battery runs within the allowable temperature for 30 cycles, both when the PCM is charged $\left(T_{\min , \mathrm{H}}\right)$ and when there is no PCM $\left(T_{\min , \mathrm{A}}\right)$. 
(a)

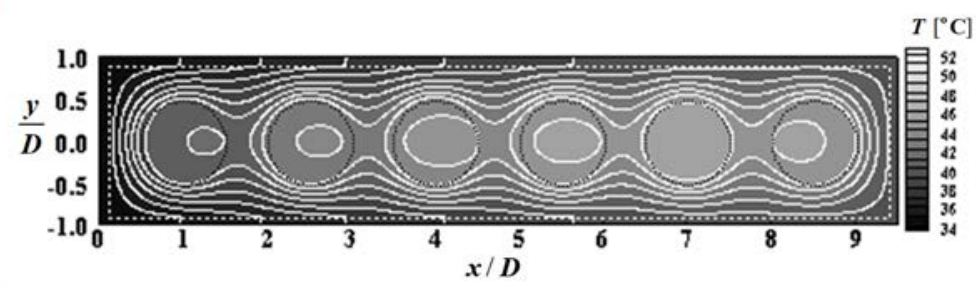

(b)

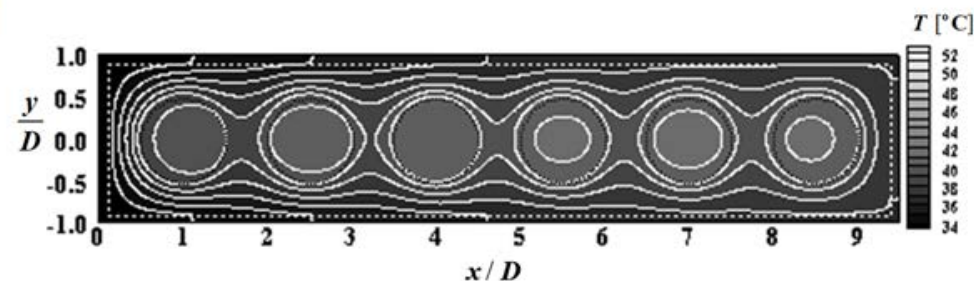

(c)

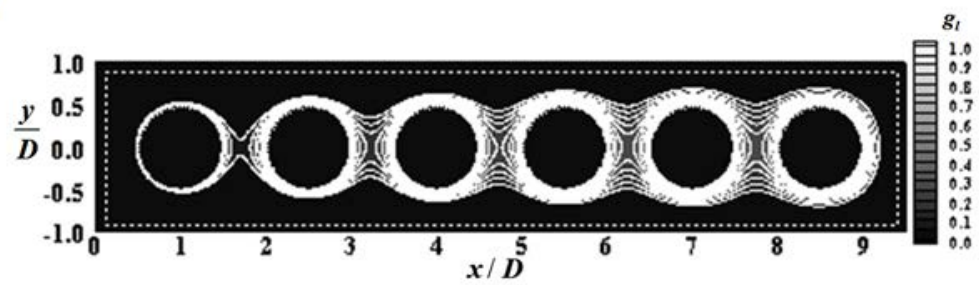

(d)

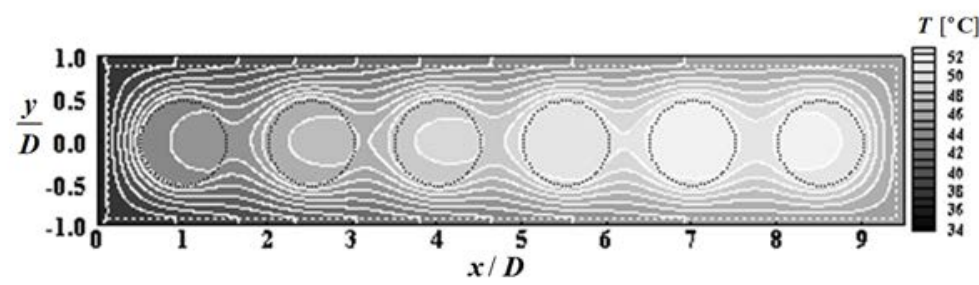

(e)

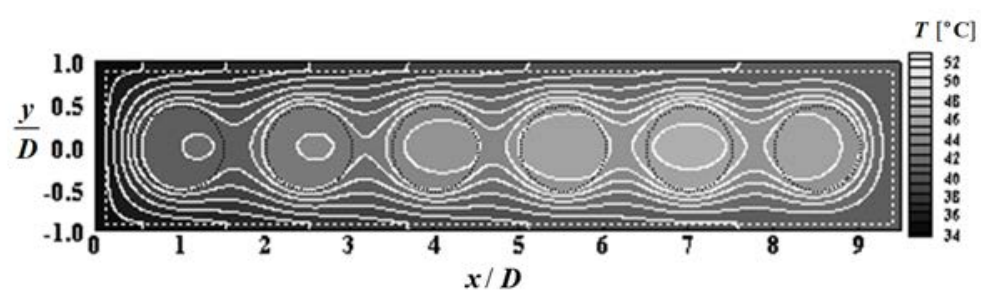

(f)

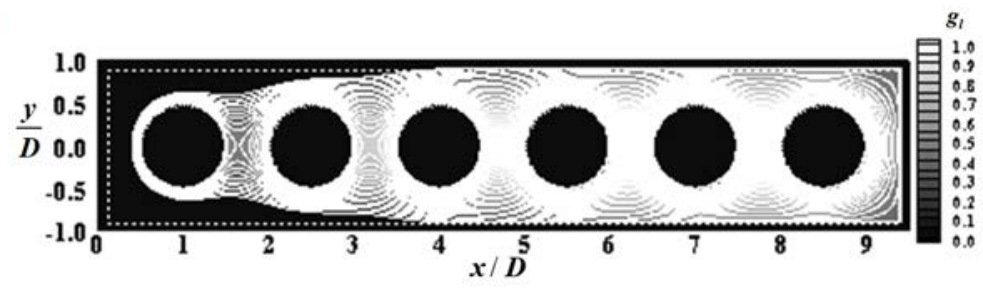

Figure 6: Temperature and liquid fraction at critical time. (a) No PCM, $t_{\mathrm{cA}, \Delta \mathrm{T}}$; (b) With PCM, $t_{\mathrm{cA}, \Delta \mathrm{T}}$; (c) Liquid fraction $\left(g_{1}\right), t_{\mathrm{cA}, \Delta \mathrm{T}}$; (d) No PCM, $t_{\mathrm{cH}, \Delta \mathrm{T}}$; (e) With PCM, $t_{\mathrm{cH}, \Delta \mathrm{T}}$; (f) $g_{1}, t_{\mathrm{cH}, \Delta \mathrm{T}}$. 


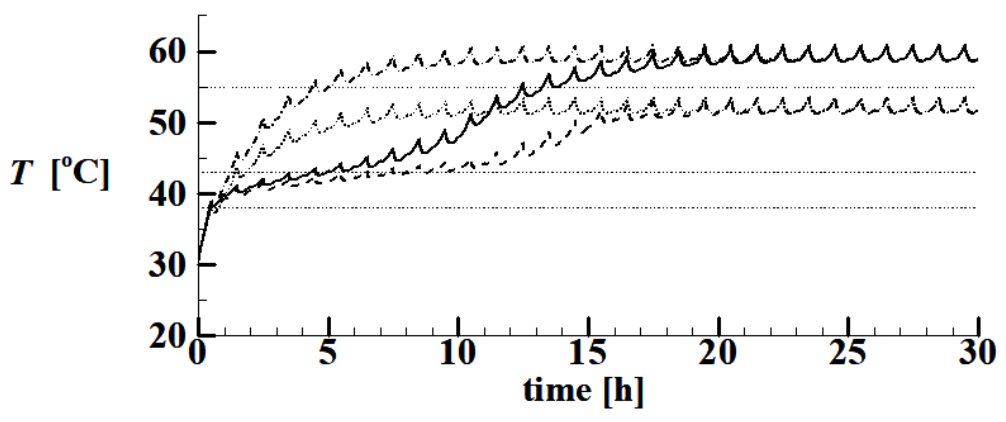

Figure 7: Transient variations of $T_{\max }$ and $T_{\min .}-T_{\max , \mathrm{H}} ;---T_{\min , \mathrm{H}} ;-\cdot-T_{\max , \mathrm{A}} ; \ldots T_{\min , \mathrm{A}}$.

After the melting completion temperature of the PCM is reached, the temperature of the battery pack filled with the phase change material approaches the temperature of the battery pack without the phase change material. After 20 charge-discharge cycles, the phase-change materials are completely melted and the temperatures of the two battery packs are almost the same. It is observed that the PCM of the design proposed in this study has an effect for 20 cycles when the charge discharge is repeated in the one hour cycle in the range of SOC 0.25 and 0.75 .

\section{CONCLUSION}

In this study, we propose a hybrid thermal management system which utilizes both active cooling using external convection cooling and passive cooling using the PCM for battery packs for hybrid vehicles. To analyze the melting process of the PCM, the immersed boundary method developed for conjugate heat transfer is extended to include the phase change process. The newly developed code was applied to the one-dimensional solidification problem and confirmed to be in good agreement with the analytical solution. The code was also validated by applying it to the battery package problem using the PCM and obtaining the result which agrees well with the FEM result found in the open literature. As a result of applying it to the hybrid thermal management system, it has been shown that charging the PCM can significantly increase the time required for the battery to reach the critical temperature from 3.6 hours to 12.3 hours. The performance of the battery thermal management system can be influenced by cooling air temperature, flow rate and current, and parametric studies regarding them will be presented in the future.

\section{REFERENCES}

[1] Bitsche, O. \& Gutmann, G., Systems for hybrid cars. Journal of Power Sources, 127(1), pp. 8-15, 2004.

[2] Khateeb, S.A., Farid, M.M., Selman, J.R. \& Al-Hallaj, S., Design and simulation of a lithium-ion battery with a phase change material thermal management system for an electric scooter. Journal of Power Sources, 128(2), pp. 292-307, 2004.

[3] Hallaj, S.A. \& Selman, J.R., A novel thermal management system for electric vehicle batteries using phase-change material. Journal of Electrochemical Society, 147(9), pp. 3231-3236, 2000.

[4] Sabbah, R., Kizilel, R., Selman, J.R. \& Al-Hallaj, S., Active (air-cooled) vs. passive (phase change material) thermal management of high power lithium-ion packs: 
Limitation of temperature rise and uniformity of temperature distribution. Journal of Power Sources, 182(2), pp. 630-638, 2008.

[5] Song, J.C., Ahn, J. \& Lee, J.S., An immersed-boundary method for conjugate heat transfer analysis. Journal of Mechanical Science and Technology, 131(5), pp. 22872294, 2017.

[6] Chung, J.D., Lee, J.S., Choi, M. \& You, H., A refined similarity solution for the multicomponent alloy solidification. International Journal of Heat and Mass Transfer, 144(13), pp. 2483-2492, 2001. 